\title{
Effect of trabeculectomy on pulsatile ocular blood flow
}

Division of Pharmacological

Sciences and Toxicology, United Medical and Dental Schools of Guy's and St Thomas's

Hospitals, St Thomas's

Hospital, London

C B James

Correspondence to:

Oxford Eye Hospital, The

Radcliffe Infirmary, Oxford OX26HE.

Accepted for publication 7 July 1994

\begin{abstract}
Trabeculectomy, despite producing an effective reduction in intraocular pressure, may not prevent continued visual field loss. This may be because of the presence of other factors in the pathogenesis of glaucoma. Vascular factors have been suggested as being particularly important. To study the effect of trabeculectomy on ocular blood flow the technique of ocular pulse analysis has been used to derive a measure of pulsatile ocular blood flow in 17 patients (average age 65.6 (SD 1.8) years) undergoing trabeculectomy. A significant increase in pulsatile ocular blood flow of $29 \%$ was observed in the group as a whole in the standing position following operation but in some individuals blood flow changed only slightly despite a large reduction in intraocular pressure. The significance of these findings in relation to the prognosis of visual field preservation following trabeculectomy is discussed. (Br F Ophthalmol 1994; 78: 818-822)
\end{abstract}

Trabeculectomy is increasingly performed in patients with chronic open angle glaucoma as it appears to be the best method of visual field preservation. ${ }^{\prime}$ None the less, continued field loss in patients with apparently satisfactory pressure control following this operation has been reported. ${ }^{2-5}$ Furthermore, a recent study suggests that reduction of intraocular pressure reduces but does not prevent the progression of glaucomatous visual damage. ${ }^{6}$ There would seem to be a number of explanations for this. Firstly, it may be that the pressure, although reduced, is still sufficiently elevated to cause damage; secondly, it may be that raised intraocular pressure triggers a pathological process which subsequent lowering of pressure fails to stop; thirdly, because glaucoma is a multifactorial disease, visual loss continues in patients with apparently satisfactory pressure control because other contributory factors remain untreated. The role of vascular factors in the pathogenesis of glaucoma has recently received much attention and there is increasing evidence that there may be either a decreased blood flow or a failure of autoregulation at the optic nerve head. ${ }^{7-9}$

Clinical investigation of ocular blood flow and its component parts is difficult. Langham and coworkers have explored the use of ocular pulse measurement to derive a measure of pulsatile ocular blood flow. ${ }^{1011}$ The ocular pulse is readily recorded with a pneumotonometer. The method essentially assesses blood flow in tissue supplied by the posterior ciliary vessels, principally the anterior optic nerve head and the choroid, the large majority of flow being through the choroid.
Central retinal artery flow accounts for only $10 \%$ of ocular blood flow.

Previous studies have shown a reduced pulsatile blood flow in patients with both chronic open angle glaucoma and low tension glaucoma. ${ }^{12} 13$ The technique has also been used to study the effect of topical $\beta$ receptor antagonist treatment on pulsatile ocular blood flow. ${ }^{12} 14$ These studies are complicated by the fact that these drugs as well as lowering intraocular pressure also produce systemic cardiovascular effects which may affect blood flow. This study using ocular pulse analysis was conducted to investigate the effect of trabeculectomy on pulsatile ocular blood flow thereby assessing the effect of reducing intraocular pressure on ocular blood flow without alteration of the systemic circulation.

\section{Methods}

\section{SUBJECTS}

Patients diagnosed as suffering from chronic open angle glaucoma in one or both eyes were recruited to the study from the glaucoma and general eye clinics at St Thomas's Hospital. A diagnosis of chronic open angle glaucoma was made if the patient had, on at least one occasion, an intraocular pressure recorded of greater than $22 \mathrm{~mm} \mathrm{Hg}$, cupping of the optic disc, and glaucomatous field loss. Gonioscopy was performed at the time of entry into the study to confirm that at least the anterior trabecular meshwork was visible; the eye was also examined to exclude pseudoexfoliation and pigmentary glaucoma. Patients judged to have severe field loss or an intraocular pressure greater than 30 $\mathrm{mm} \mathrm{Hg}$ on treatment were not admitted to the study. Providing these criteria were met previous medical and laser therapy was not a bar to entry into the study nor was previous surgery of any sort to the control eye.

Thirteen men and seven women about to undergo trabeculectomy were initially entered into the study. One female patient withdrew from the study before operation. One male patient was withdrawn from the study following operation as it was uncertain whether or not he was following the study protocol for cessation of topical treatment before measurement. One female patient had to be withdrawn from the study as the intraocular pressure rose by more than $10 \mathrm{~mm} \mathrm{Hg} 5$ days after stopping treatment. The mean age of the remaining group was 65.6 (SD 1.8) years. The refractive error of the operated eye was $+0.4(0.3)$ dioptres and of the unoperated eye $+0.75(0.4)$ dioptres.

The effect of ocular surgery on the eye was controlled for by examining a group of patients 
without glaucoma or other ocular disease about to undergo cataract extraction and lens implantation. The measurement protocol used in this group was identical to that used in the trabeculectomy patients. Ten subjects, six male and four female, were examined. The mean age of the group was $67.6(3.4)$ years. The refractive error of the operated eye was $-2 \cdot 5(1 \cdot 2)$ dioptres and of the unoperated eye $-1 \cdot 3(1 \cdot 2)$ dioptres.

STUDY PROTOCOL

Ethical approval for this study was obtained from the local ethics committee. The following protocol was observed on all patients entering the study. Initial assessment was made with the patient on topical treatment. A full history was taken to include systemic medications (Table 1). Visual acuity and refractive error were measured and slit-lamp examination performed followed by gonioscopy and funduscopy. Topical treatment was suspended for 14 days in both eyes, at the end of which ocular pulse measurements were made with a pneumotonometer connected to a Langham ocular blood flow system in the standing position after 5 minutes' rest and lying position after 10 minutes' rest under oxybuprocaine $0.4 \%$ anaesthesia. Four 5 second measurements were made in each eye recording from the right eye first and the results averaged. Heart rate was recorded using an ECG and an autosphygmomanometer was used to measure blood pressure twice in each posture. Mean blood pressure was calculated as diastolic pressure plus one third systemic pulse pressure. Pulsatile ocular blood flow was calculated as previously described..$^{1113}$ Patients on triple therapy reduced their medication by one therapy a day. No patient was on carbonic anhydrase inhibitors. The intraocular pressure was measured at 5 days following suspension of treatment; if the pressure had risen by more than $10 \mathrm{~mm} \mathrm{Hg}$ the patient was withdrawn from the study and treatment immediately recommenced. Follow-

Table 1 Systemic medications of patients involved in the study

\begin{tabular}{|c|c|c|c|c|c|c|}
\hline Patient & Age & Sex & $\begin{array}{l}\text { Calcium } \\
\text { antagonist }\end{array}$ & $\begin{array}{l}\beta \text { Receptor } \\
\text { antagonist }\end{array}$ & Diuretic & Other \\
\hline \multicolumn{7}{|c|}{ Trabeculectomy patients: } \\
\hline 1 & 68 & M & $\star$ & $\star$ & & Chlorthalidone \\
\hline 2 & 69 & M & & & & \\
\hline 3 & 59 & $M$ & & & & \\
\hline 4 & 66 & M & & & & \\
\hline 5 & 68 & $\mathrm{~F}$ & & & & \\
\hline $\begin{array}{l}6 \\
7\end{array}$ & $\begin{array}{l}74 \\
59\end{array}$ & $M$ & $\star$ & $\star$ & ^ & Warfarin, amiodarone, enalapril \\
\hline 8 & 54 & $\stackrel{M}{F}$ & & & & \\
\hline 9 & 65 & $\mathbf{M}$ & $\star$ & $\star$ & & \\
\hline 10 & 69 & $M$ & & & & \\
\hline 11 & 65 & $\mathbf{M}$ & & $\star$ & & Ibuprofen \\
\hline 12 & 58 & $M$ & & & & \\
\hline 13 & 76 & $\mathbf{M}$ & * & & & \\
\hline 14 & 74 & $M$ & & $\star$ & & Hydralazine, metformin \\
\hline 15 & 66 & $\mathbf{F}$ & & & & Captopril \\
\hline 16 & 75 & $\mathrm{~F}$ & & $\star$ & & \\
\hline \multirow{2}{*}{\multicolumn{7}{|c|}{ Cataract patients: }} \\
\hline & & & & & & \\
\hline 1 & & M & & & & Metformin \\
\hline 2 & 71 & $M$ & & & & Minocycline \\
\hline 3 & 66 & $\mathbf{M}$ & & & & \\
\hline $\begin{array}{l}4 \\
5\end{array}$ & 75 & $M$ & ^ & & $\star$ & Glyceryl trinitrate \\
\hline 6 & 78 & $M$ & & & & \\
\hline 7 & 54 & $\mathrm{~F}$ & & $\star$ & $\star$ & Glibenclamide \\
\hline 8 & 84 & $\mathrm{~F}$ & & & & Prochlorperazine \\
\hline 9 & 74 & $\mathbf{M}$ & & & & \\
\hline 10 & 72 & $\mathbf{F}$ & & ^ & & Metformin \\
\hline
\end{tabular}

ing pulse measurements at 14 days, topical treatment was recommenced. The patients subsequently underwent routine trabeculectomy. Assessment was again performed at 3 and 6 months after surgery and ocular pulse studies again performed in both standing and lying positions. Topical treatment to the fellow eye was stopped on each occasion 14 days before measurement. No patient was on treatment for the operated eye at this time. Measurements and calculations were not possible on some patients at points during the study as their intraocular pressure fell below $10 \mathrm{~mm} \mathrm{Hg}$ : Calculation of pulsatile ocular blood flow could not be made in these cases as no data are available for the conversion of the pressure pulse to a volume pulse below $10 \mathrm{~mm} \mathrm{Hg}$. The patients excluded for this reason are detailed in the tables of results. This may introduce a bias into the results. Measurements in individual patients were always made at the same time of day throughout the study. No patient underwent any change in systemic medication.

\section{Statistical analysis}

Paired $t$ tests and Wilcoxon signed rank nonparametric tests were used to examine the postural differences in the patients. The results of the tests were similar and those of the $t$ tests are given. Repeat measure analysis of variance was used to examine the group for changes following trabeculectomy.

Both within, between occasion, and between eye reproducibility were assessed in 10 healthy volunteers to determine the coefficient of variation of the technique in measuring intraocular pressure, ocular pulse amplitude, pulsatile ocular blood flow, and heart rate. The technique employed in the main study was employed in the ocular pulse measurements. The within occasion coefficient of variation for pulsatile ocular blood flow measurement was $10 \%$ standing and $10 \cdot 8 \%$ lying: for between occasions the results were $15 \cdot 7 \%$ and $18 \cdot 4 \%$ respectively (Table 2 ).

\section{Results}

\section{EFFECT OF TRABECULECTOMY ON THE OCULAR} PULSE

As expected in the trabeculectomy patients there was a marked, highly significant reduction in intraocular pressure in both standing (45\%) and lying $(48 \%)$ positions at 3 and 6 months after trabeculectomy. In the standing position this

Table 2 Coefficients of variation (\%) for within occasion, between two separate occasions, and between eyes for intraocular pressure, pulse amplitude, pulsatile ocular blood flow, and heart rate in the standing and lying postures $(n=10)$

\begin{tabular}{lccc}
\hline & $\begin{array}{l}\text { Within } \\
\text { occasion }\end{array}$ & $\begin{array}{l}\text { Between } \\
\text { occasions }\end{array}$ & $\begin{array}{l}\text { Between } \\
\text { eyes }\end{array}$ \\
\hline Intraocular pressure standing & $6 \cdot 8$ & $8 \cdot 4$ & $4 \cdot 1$ \\
Intraocular pressure lying & 6.9 & 12.9 & 3.9 \\
Pulse amplitude standing & $11 \cdot 2$ & $15 \cdot 2$ & $5 \cdot 7$ \\
Pulse amplitude lying & $9 \cdot 2$ & 12.6 & $5 \cdot 4$ \\
Pulsatile ocular blood flow standing & $10 \cdot 0$ & $15 \cdot 7$ & $7 \cdot 5$ \\
Pulsatile ocular blood flow lying & $10 \cdot 8$ & $18 \cdot 4$ & $7 \cdot 1$ \\
Heart rate standing & 3.8 & 3.6 & \\
Heart rate lying & 3.3 & $4 \cdot 0$ & \\
\hline
\end{tabular}


was associated with no significant decrease in the pulse amplitude at 3 months but pulsatile ocular blood flow increased significantly $(95$ (36) $\mu \mathrm{l} / \mathrm{min}$, $31 \%$ of the preoperative value). There was a significant decrease in the pulse amplitude at 6 months of $0.4(0.2) \mathrm{mm} \mathrm{Hg}$ and a significant increase in the pulsatile ocular blood flow of 91 (46) $\mu \mathrm{l} / \mathrm{min}$ ( $29 \%$ of the preoperative value). Systemic variables of heart rate, mean blood pressure, and systemic pulse pressure remained unchanged during the examination period (Table 3).

In the lying position pulse amplitude was significantly reduced by $31 \%$ following trabeculectomy but pulsatile ocular blood flow was virtually unchanged from the preoperative value (Table 3).

No significant changes in intraocular pressure or ocular pulse variables were recorded in the unoperated eye in either the standing or the lying position during the course of the study (Table 3 ).

Using the coefficient of variation calculations the between occasion standard deviation for preoperative pulsatile ocular blood flow in the standing patients with glaucoma should be 55.6 $\mu \mathrm{l} / \mathrm{min}$. The mean postoperative difference in blood flow was $93 \mu \mathrm{l} / \mathrm{min}$. This gives the standing results a power of $98 \%$ with a two sided significance of $0 \cdot 05$.

EFFECT OF TRABECULECTOMY ON POSTURAL CHANGES IN THE OCULAR PULSE

A significant postural increase in intraocular pressure on lying occurred both before $(4 \cdot 8(0.5)$ $\mathrm{mm} \mathrm{Hg})$ and after surgery $(2 \cdot 8(0 \cdot 8) \mathrm{mm} \mathrm{Hg}$ at 6 months), (Table 4). Measurement of postural change in pulsatile ocular blood flow reveals a slight increase on lying down before operation although this did not reach statistical significance. Following surgery a significant decrease in pulsatile ocular blood flow was seen on lying down, 77 (15) $\mu \mathrm{l} / \mathrm{min}$ at 3 months and 31 (14) $\mu \mathrm{l} / \mathrm{min}$ at 6 months (Table 4). No postural change in pulsatile ocular blood flow was seen in the control eye of the patients undergoing trabeculectomy. This was a diverse group of eyes, however, some had had previous glaucoma surgery and others not. There was a significant fall in mean blood pressure and heart rate in the supine posture (Table 4). No statistically significant difference between the pre and postoperative postural changes in mean blood pressure and heart rate was detected.

\section{EFFECT OF CATARACT SURGERY ON THE OCULAR} PULSE

Following surgery there was a significant reduction in intraocular pressure by $3.9(0.9) \mathrm{mm} \mathrm{Hg}$ at 3 months postoperatively in the standing position and $3.3(1.2) \mathrm{mm} \mathrm{Hg}$ in the lying position in the operated eye only. The change was smaller at 6 months $(1.8(0.7) \mathrm{mm} \mathrm{Hg}$ and $1.8(0.9) \mathrm{mm} \mathrm{Hg}$ respectively). In the lying position this just failed to reach statistical significance. No change in the ocular pulse or its derivatives in either the standing or the lying position occurred (Table 5). There were no changes in the systemic parameters of pulse rate, mean blood pressure and systemic pulse pressure throughout the study (Table 5).

The postural changes in intraocular pressure and pulsatile ocular blood flow are shown in Table 6. A significant increase in intraocular pressure is seen together with a significant fall in pulsatile ocular blood flow in the supine posture. These changes are associated with a fall in blood

Table 3 Change in the standing and lying ocular and systemic measurements in the operated and unoperated eye after trabeculectomy $(S E M)$. (Operated standing $n=12 ;$ unoperated standing $n=15$; operated lying $n=16 ;$ unoperated lying $n=17$ )

\begin{tabular}{|c|c|c|c|c|c|c|}
\hline \multirow[b]{2}{*}{ Variable } & \multirow[b]{2}{*}{$\begin{array}{l}\text { Before } \\
\text { surgery }\end{array}$} & \multicolumn{2}{|l|}{ Operated eye } & \multirow[b]{2}{*}{$\begin{array}{l}\text { Before } \\
\text { surgery }\end{array}$} & \multicolumn{2}{|c|}{ Unoperated eye } \\
\hline & & $\begin{array}{l}3 \text { Months } \\
\text { after surgery }\end{array}$ & $\begin{array}{l}6 \text { Months } \\
\text { after surgery }\end{array}$ & & $\begin{array}{l}3 \text { Months } \\
\text { after surgery }\end{array}$ & $\begin{array}{l}6 \text { Months } \\
\text { after surgery }\end{array}$ \\
\hline \multicolumn{7}{|l|}{ Standing: } \\
\hline Intraocular pressure $(\mathrm{mm} \mathrm{Hg})$ & $27 \cdot 8(1 \cdot 9)$ & $15 \cdot 2(1 \cdot 1)^{\star}$ & $15 \cdot 3(1 \cdot 0)^{\star}$ & $23 \cdot 6(1 \cdot 5)$ & $24(1 \cdot 8)$ & $23.4(1.6)$ \\
\hline Pulse amplitude (mm Hg) & $1.9(0 \cdot 1)$ & $1.7(0.2)$ & $1.5(0 \cdot 2)^{\star}$ & $1 \cdot 8(0 \cdot 1)$ & $1 \cdot 8(0 \cdot 1)$ & $1.7(0.2)$ \\
\hline Pulsatile ocular blood flow $(\mu \mathrm{l} / \mathrm{min})$ & $310(33)$ & $405(37)^{\star}$ & $401(65)^{\star}$ & $345(38)$ & $307(21)$ & $302(37)$ \\
\hline Blood pressure $(\mathrm{mm} \mathrm{Hg})$ & $111(5)$ & $111(6)$ & $113(7)$ & $109(4)$ & $106(4)$ & $108(5)$ \\
\hline Systemic pulse pressure $(\mathrm{mm} \mathrm{Hg})$ & $57(3)$ & $59(7)$ & $56(6)$ & $54(5)$ & $55(5)$ & $54(4)$ \\
\hline Heart rate $(\mathrm{bpm})$ & $72(3)$ & $67(4)$ & $72(4)$ & $72(3)$ & $65(3)$ & $72(3)$ \\
\hline \multicolumn{7}{|l|}{ Lying: } \\
\hline Intraocular pressure $(\mathrm{mm} \mathrm{Hg})$ & $33.0(1 \cdot 8)$ & $16 \cdot 8(1 \cdot 0)^{\star}$ & $17 \cdot 3(1 \cdot 0)^{\star}$ & $28 \cdot 4(1 \cdot 9)$ & $25 \cdot 7(2 \cdot 0)$ & $24 \cdot 6(2 \cdot 0)$ \\
\hline Pulse amplitude (mm Hg) & $2 \cdot 5(0 \cdot 2)$ & $1 \cdot 6(0 \cdot 1)^{\star}$ & $1 \cdot 6(0 \cdot 2)^{\star}$ & $2 \cdot 1(0 \cdot 1)$ & $2 \cdot 1(0 \cdot 1)$ & $2 \cdot 0(0 \cdot 2)$ \\
\hline Pulsatile ocular blood flow $(\mu \mathrm{l} / \mathrm{min})$ & $333(29)$ & $330(30)$ & $348(43)$ & $330(33)$ & $313(27)$ & $328(39)$ \\
\hline Blood pressure $(\mathrm{mm} \mathrm{Hg})$ & $99(3)$ & $100(3)$ & $100(4)$ & $98(32)$ & $99(3)$ & $99(4)$ \\
\hline Systemic pulse pressure $(\mathrm{mm} \mathrm{Hg}$ ) & $53(4)$ & $55(4)$ & $58(4)$ & $53(3)$ & $54(4)$ & $57(4)$ \\
\hline Heart rate $(\mathrm{bpm})$ & $63(3)$ & $61(3)$ & $63(3)$ & $65(3)$ & $61(3)$ & $63(3)$ \\
\hline
\end{tabular}

$\star$ Significant Fischer PLSD test at $95 \%$ or more.

Table 4 Postural change in intraocular pressure and pulsatile ocular blood flow (POBF) before and after trabeculectom $(S E M)$ ( $n=17$ for intraocular pressure measurement, $n=17$ for pulsatile ocular blood flow presurgery measurement, $n=14$ for pulsatile ocular blood flow $3 / 12$ post-surgery measurement, $n=15$ for pulsatile ocular blood flow $6 / 12$ post-surgery measurement)

\begin{tabular}{|c|c|c|c|c|c|c|c|c|c|}
\hline \multirow[b]{2}{*}{ Variable } & \multicolumn{3}{|c|}{ Before surgery } & \multicolumn{3}{|c|}{3 Months after surgery } & \multicolumn{3}{|c|}{6 Months after surgery } \\
\hline & Change & $\mathbf{t}$ & $p$ Value & Change & $\mathbf{t}$ & p Value & Change & $\mathbf{t}$ & $p$ Value \\
\hline $\begin{array}{l}\text { Intraocular pressure }(\mathrm{mm} \mathrm{Hg}) \\
\text { Pulsatile ocular blood flow }(\mu \mathrm{l} / \mathrm{min}) \\
\text { Blood pressure (mm Hg) } \\
\text { Systemic pulse pressure }(\mathrm{mm} \mathrm{Hg}) \\
\text { Heart rate }(\mathrm{bpm})\end{array}$ & $\begin{array}{l}4 \cdot 8(0 \cdot 5) \\
29(16) \\
11 \cdot 6(1 \cdot 8) \\
3 \cdot 6(2 \cdot 1) \\
9 \cdot 2(1 \cdot 5)\end{array}$ & $\begin{array}{l}9 \cdot 8 \\
1.8 \\
6 \cdot 6 \\
1.7 \\
6 \cdot 0\end{array}$ & $\begin{array}{l}0.0001 \\
\text { NS } \\
0 \cdot 001 \\
\text { NS } \\
0 \cdot 0001\end{array}$ & $\begin{array}{r}2 \cdot 9(0 \cdot 4) \\
-77(15) \\
9 \cdot 2(2 \cdot 0) \\
2 \cdot 9(2 \cdot 0) \\
6 \cdot 8(1 \cdot 0)\end{array}$ & $\begin{array}{c}7 \cdot 6 \\
-5 \\
4 \cdot 5 \\
1 \cdot 4 \\
6 \cdot 9\end{array}$ & $\begin{array}{l}0.0001 \\
0.0003 \\
0.0003 \\
\text { NS } \\
0.0001\end{array}$ & $\begin{array}{r}2 \cdot 8(0 \cdot 8) \\
-31(14) \\
10 \cdot 3(1 \cdot 8) \\
-0 \cdot 4(1 \cdot 5) \\
7 \cdot 6(1 \cdot 6)\end{array}$ & $\begin{array}{c}3 \cdot 4 \\
-2 \cdot 1 \\
5 \cdot 9 \\
0 \cdot 28 \\
4 \cdot 8\end{array}$ & $\begin{array}{l}0.003 \\
0.05 \\
0.0001 \\
\text { NS } \\
0.002\end{array}$ \\
\hline
\end{tabular}

NS $=$ Not significant 
Table 5 Change in the standing and lying ocular and systemic measurements in the operated and unoperated eye following cataract extraction $(S E M)(n=10)$

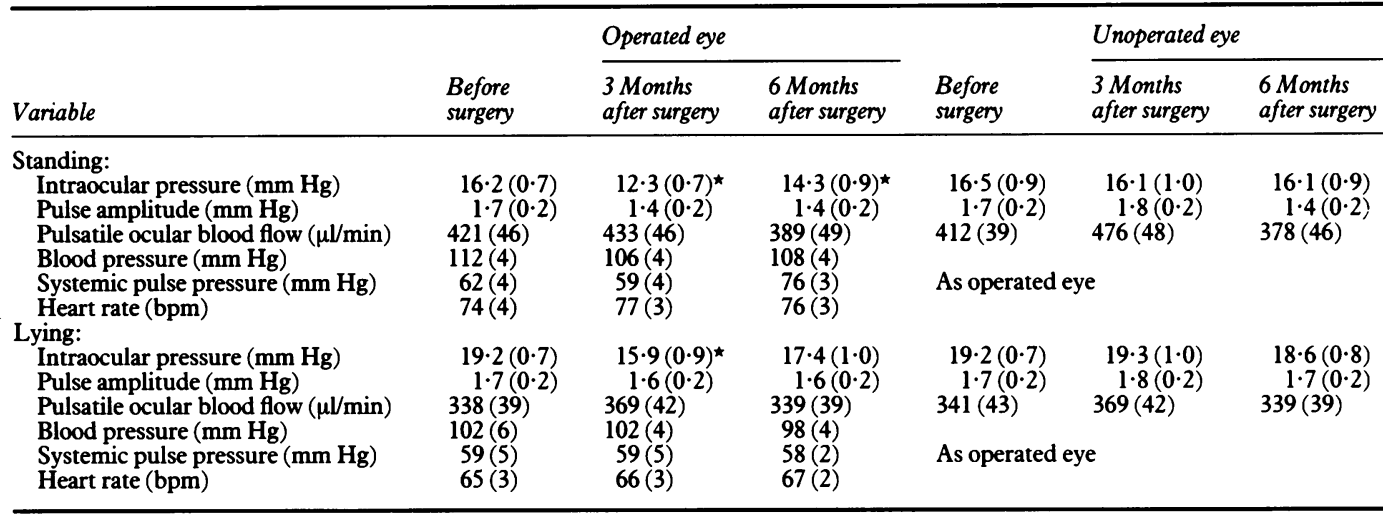

${ }^{\star}$ Significant Fischer PLSD test at $95 \%$ or more.

Table 6 Postural change in intraocular pressure and pulsatile ocular blood flow before and after uncomplicated cataract extraction $(S E M)(n=10)$

\begin{tabular}{|c|c|c|c|c|c|c|c|c|c|}
\hline \multirow{2}{*}{ Variable } & \multicolumn{3}{|l|}{ Before surgery } & \multicolumn{3}{|c|}{3 Months after surgery } & \multicolumn{3}{|c|}{6 Months after surgery } \\
\hline & Change & $\mathbf{t}$ & $p$ Value & Change & $\mathrm{t}$ & $p$ Value & Change & $\mathrm{t}$ & p Value \\
\hline $\begin{array}{l}\text { Intraocular pressure }(\mathrm{mm} \mathrm{Hg}) \\
\text { Pulsatile ocular blood flow }(\mu \mathrm{l} / \mathrm{min}) \\
\text { Blood pressure }(\mathrm{mm} \mathrm{Hg}) \\
\text { Systemic pulse pressure }(\mathrm{mm} \mathrm{Hg}) \\
\text { Hear rate (bpm) }\end{array}$ & $\begin{array}{r}3 \cdot 0(0 \cdot 4) \\
-83(18) \\
4 \cdot 9(1 \cdot 2) \\
3 \cdot 3(4 \cdot 1) \\
8 \cdot 7(2 \cdot 8)\end{array}$ & $\begin{array}{r}6 \cdot 9 \\
-4 \cdot 5 \\
4 \cdot 1 \\
0 \cdot 8 \\
3 \cdot 1\end{array}$ & $\begin{array}{l}0.0001 \\
0.001 \\
0.003 \\
\mathrm{NS} \\
0.01\end{array}$ & $\begin{array}{c}3 \cdot 6(0 \cdot 5) \\
-64(38) \\
2 \cdot 2(2 \cdot 9) \\
-0 \cdot 8(3) \\
10 \cdot 7(2 \cdot 4)\end{array}$ & $\begin{array}{c}7 \cdot 2 \\
-1 \cdot 7 \\
0 \cdot 77 \\
0 \cdot 27 \\
4 \cdot 4\end{array}$ & $\begin{array}{l}0.0001 \\
\text { NS } \\
\text { NS } \\
\text { NS } \\
0.002\end{array}$ & $\begin{array}{c}3 \cdot 1(0 \cdot 5) \\
-50(19) \\
4 \cdot 4(2 \cdot 6) \\
0 \cdot 4(4) \\
8 \cdot 8(1 \cdot 8)\end{array}$ & $\begin{array}{r}6 \cdot 2 \\
-6 \cdot 2 \\
1 \cdot 7 \\
0 \cdot 1 \\
4 \cdot 8\end{array}$ & $\begin{array}{l}0.0002 \\
0.03 \\
\mathrm{NS} \\
0.001 \\
0.001\end{array}$ \\
\hline
\end{tabular}

NS $=$ Not significant

pressure and heart rate (Table 6). The fall in blood pressure did not reach statistical significance postoperatively.

\section{Discussion}

The effect that surgery has on the generation of the ocular pulse must first be discussed before considering the effect of trabeculectomy on pulsatile ocular blood flow. No significant change in pulsatile ocular blood flow following surgery was observed in patients undergoing cataract extraction although there was a small but significant reduction in intraocular pressure in the operated eye. This suggests that intraocular surgery itself, particularly healing and fibrosis of a wound which might be expected to result in a change in ocular rigidity, does not significantly alter the characteristics of the ocular pulse. There are, however, obvious differences between the two operations. The presence of a drainage bleb may theoretically dampen down the ocular pulse thus reducing the estimate of pulsatile ocular blood flow. It is interesting in this respect that, as in another study, ${ }^{15}$ the postural increase in intraocular pressure on assuming the supine posture persisted postoperatively. This suggests that a filtering operation, although lowering the overall level of intraocular pressure does not alter the response of the eye to physiological manoeuvres that alter intraocular pressure. As this is true for the postural change in intraocular pressure it is likely that it is also true for the much faster pulsatile elevations in intraocular pressure during the cardiac cycle. It also appears that any induction of changes in corneal curvature following the two surgical procedures does not significantly alter the ocular pulse. Furthermore, changes in corneal curvature following trabeculectomy appear slight. ${ }^{16}$

In a longitudinal study changes in systemic variables (heart rate, mean blood pressure, and systemic pulse pressure) must also be considered. No significant change occurred in these variables. Additionally, there was no significant change in the ocular pulse and its derivatives in the unoperated eye. This would be expected for no significant change in intraocular pressure was seen throughout the study in the unoperated eye.

It is probably better to think of the calculated pulsatile ocular blood flow produced using this technique as an index of pulsatile blood flow rather than an absolute measure; they may, for example, be affected by variables that are not associated with ocular blood flow such as the axial length of the eye. ${ }^{17}$ The technique measures only pulsatile flow and the proportion of pulsatile flow to total flow is unknown and may vary. ${ }^{18} \mathrm{An}$ increase in the proportion of non-pulsatile flow would be anticipated as intraocular pressure fell. Measurements should, however, have validity in a longitudinal study in which systemic variables are constant, and the ocular dimensions (axial length) unchanged.

The increase in pulsatile ocular blood flow in the standing position following trabeculectomy would thus appear to be of significance. Trabeculectomy is associated with a large decrease in intraocular pressure. If no autoregulation exists this causes an increase in perfusion pressure which will increase both pulsatile and nonpulsatile flow. This would appear to be the situation in the standing posture. In the lying posture a reduction in pulsatile ocular blood flow compared with the standing measurements has previously been reported in patients with chronic open angle glaucoma and low tension 
glaucoma. ${ }^{12} 13$ This was not seen in the patients with chronic open angle glaucoma before operation in this study, .who had a much higher intraocular pressure than seen in these previously studied groups. This may indicate that a degree of autoregulation occurs in the choroidal circulation at elevated intraocular pressure to prevent the already reduced pulsatile ocular blood flow falling further with a change in posture. Thus when intraocular pressure is lowered in this posture no change in pulsatile ocular blood flow would be expected, as indeed was the case here. Following surgery, however, the usual postural change in pulsatile blood flow is regained. Changes in systemic mean blood pressure and heart rate were not significantly different pre and postoperatively and thus will not contribute to this change in postural response. The presence of autoregulation in the choroidal circulation is a matter of debate but some experimental animal studies suggest that it may occur. ${ }^{19}$

The pulsatile ocular blood flow did not increase in all patients following trabeculectomy, however. In some patients there was no change in pulsatile ocular blood flow following surgery while in others a decrease was observed despite an appreciable fall in intraocular pressure. This occurred in four patients in the standing position and eight in the lying position. In only one patient did intraocular pressure fail to fall following trabeculectomy; little change in pulsatile ocular blood flow in either posture was seen in this patient. Care must be taken in the interpretation of individual results, not least because of the between occasion coefficient of variation of the technique. Furthermore, it has been suggested that autoregulation may occur in the lying posture which will influence the change seen in pulsatile ocular blood flow following a reduction in intraocular pressure. It is interesting, however, that a reduction in intraocular pressure does not always ensure that progression of glaucomatous damage is prevented..$^{2-5}$ If it is the patients whose pulsatile ocular blood flow fails to increase following successful surgery who continue to develop visual loss this would provide support for the hypothesis of Schulzer et $a l^{20}$ that vascular factors may have a part in the pathogenesis of a subset of patients with chronic open angle glaucoma.

The increase in pulsatile ocular blood flow in the erect posture seen in patients undergoing trabeculectomy may be a factor in the apparent advantage the treatment has over some medical forms of glaucoma treatment. ${ }^{1}$ No increase in pulsatile ocular blood flow was seen in patients on topical timolol. ${ }^{12}$ A direct comparison between the two studies must be made with care. The initial intraocular pressure in the patients treated with timolol was lower than that reported here and the pressure reduction was less than $50 \%$ of that seen here $(4.4 \mathrm{~mm} \mathrm{Hg}$ standing in the patients treated with timolol, $12.6 \mathrm{~mm} \mathrm{Hg}$ in patients following trabeculectomy). A statistically significant increase in pulsatile ocular blood flow of $13 \%$ has been reported following single topical application of levobunolol where a reduction in intraocular pressure of $8.2 \mathrm{~mm} \mathrm{Hg}$ was observed. ${ }^{14}$ It may be that the technique of ocular pulse analysis is not sensitive enough to measure changes in pulsatile ocular blood flow associated with a small change in intraocular pressure.

\section{Conclusion}

Trabeculectomy increases pulsatile ocular blood flow in the standing posture but has no effect on the lying measurement of pulsatile ocular blood flow. This postural difference may be explained by the finding that in the preoperative untreated subjects the postural reduction in pulsatile ocular blood flow reported in previous studies was not observed. This postural change in pulsatile ocular blood flow appears to be related to intraocular pressure. At high intraocular pressures pulsatile ocular blood flow is similar in the erect and supine postures; following trabeculectomy the more usual reduction in pulsatile ocular blood flow in the supine posture is seen. It is hypothesised that the observed increase in standing pulsatile ocular blood flow in the group as a whole following trabeculectomy, if reflecting events at the optic nerve head, may be an important factor in the success of trabeculectomy in reducing glaucomatous visual damage.

I would like to thank Professor S E Smith for his great help and encouragement during this study and the consultant ophthalmologists at St Thomas's hospital for permission to examine their patients. This research was supported by the Special Trustees of $S t$ patients. This research was supported by the Special Trustees of St
Thomas's Hospital and the Royal National Institute for the Blind.

1 Jay JL, Murray SB. Early trabeculectomy versus conventiona management in primary open angle glaucoma. $\mathrm{Br} \mathcal{F}$ Ophthalmol 1988; 72: 881-9.

2 Jerndal T, Lundstrom $M$. Trabeculectomies - a follow-up study through 1/2-3 years. Acta Ophthalmol 1977; 55: 52-61.

3 Werner EB, Drance SM, Schulzer M. Trabeculectomy and the progression of glaucomatous visual field loss. Arch progression of glaucomato

4 Werner EB, Drance SM. Progression of glaucomatous field defects despite successful filtration. Can $\mathcal{F}$ Ophthalmol 1977 12: $275-80$.

5 Watson PG, Jakeman C, Ozturk M, Barnett MF, Barnett F Khaw KT. The complications of trabeculectomy (a twenty year follow-up). Eye 1990; 4: 425-38.

6 Jay LJ, Murdoch JR. The rate of visual field loss in untreated open angle glaucoma. Br f Ophthalmol 1993; 77: 176-8.

7 Drance SM. Bowman lecture. Glaucoma - changing concepts. Eye 1992; 6: 337-45.

8 Hitchings RA. Glaucoma - a modern view [Editorial]. Eye 1992; 6: 336 .

9 Van Buskirk EM, Cioffi GA. Glaucomatous optic neuropathy. Am $\mathcal{F}$ Ophthalmol 1992; 112: 447-52.

10 Langham ME, Farrell RA, O'Brien V, Silver DM, Schilder P. Blood flow in the human eye. In: Hopkins SD, ed. Ocular blood flow. Acta Ophthalmol (suppl) 1989; 191: 9-13.

11 Silver DM, Farrell RA, Langham ME, O'Brien V, Schilder P. Estimation of pulsatile ocular blood flow from intraocular pressure. In: Hopkins SD, ed. Ocular blood flow. Acta Ophthalmol (suppl) 1989; 191: 25-9.

12 Trew DR, Smith SE. Postural studies in pulsatile ocular bloodflow: II Chronic open angle glaucoma. BrfOphthalmol 1991; 75: 71-5.

13 James CB, Smith SE. Pulsatile ocular blood flow in patients with low tension glaucoma. Br F Ophthalmol 1991; 75: 46670.

14 Bosem ME, Lusky M, Weinreb RN. Short term effects of Levobunolol on ocular pulsatile flow. Am $\mathcal{f}$ Ophthalmo 1991; 114: 280-6

15 Parsley J, Powell RG, Keightley SJ, Elkington AR. Postura response of intraocular pressure in chronic open angle glaucoma following trabeculectomy. Br $\mathcal{F}$ Ophthalmol 1987; 71: 494-6.

16 Hugkulstone $\mathrm{CE}$. Changes in keratometry following trabeculectomy. Br f Ophthalmol 1991; 75: 217-8.

17 James CB, Trew DR, Clark K, Smith SE. Factors influencing the ocular pulse-axial length. Graefes Arch Clin Exp Ophthalmol 1991; 229: 341-4.

18 Trew DR, James CB, Thomas SHL, Sutton R, Smith SE Factors influencing the ocular pulse - the heart rate. Graefes Arch Clin Exp Ophthalmol 1991; 229: 553-6.

$19 \mathrm{Kiel}$ JW, Shepherd AP. Autoregulation of choroidal bloodflow in the rabbit. Invest Ophthalmol Vis Sci 1992; 33: 2399-410.

20 Schulzer M, Drance SM, Carter CJ, Brooks DE, Doublas GR, Lau W. Biostatistical evidence for two distinct chronic open angle glaucoma populations. Br f Ophthalmol 1990; 74: 196 200. 